\title{
Hacking als Widerstand
}

Was steckt hinter der Kraft und der Reichweite von Überraschungsereignissen? Ein erster Grund für sie ist die Unregierbarkeit von Prozessen. Sie erschüttern die globale Welt über neue Ressourcen. Zweitens ergibt sie sich aus der Opposition gegenüber transnationalen Unternehmen. Diese halten sich selbst für neue öffentliche Organismen, die Gouvernementalität auf den Märkten generieren. An dritter Stelle steht die grenzüberschreitende Imagination der Ausgegrenzten, die die institutionalisierten Strukturen umgeht.

Uns als Bürger neu zu erfinden bedeutet, Verhaltensweisen gegenüber den Institutionen der Bildung, der Familie und der Religion unter Einbindung neuer Technologien zu konstruieren. Dabei unterscheiden sich unsere gegenwärtigen Verhaltensweisen von denjenigen, die uns innerhalb der bisherigen Strukturen einen stabilen Sinn verliehen hatten. Kreative junge Menschen werden beispielsweise zu Unternehmern und wenden das Wissen, das sie an der Universität erworben haben, neu an. Dadurch arbeiten sie außerhalb von Betrieben und staatlichen Institutionen und werden stattdessen Teil assoziativer Produktionsnetzwerke, NGOs oder außerparteiischer politischer Bewegungen. Die Ziele dieser Organisationen sind häufig die Stärkung des sozialen Gefüges und der Sicherheit der Bürger, sowie die Zusammenarbeit bei der Gemeindeentwicklung und der Einsatz für Geschlechterparität.

Viele Modifikationen des vermittelten oder erlernten Wissens haben bereits eine gewisse Ähnlichkeit zum Hacking. Diese Modifikationen des Wissens entstehen beispielsweise im Kontext der Migration oder bei Tätigkeiten, auf die die Schule Individuen nicht vorbereitet. Objekte und Informationen werden zweckentfremdet, erhalten eine neue Bedeutung, werden Teil eines neuen Codes oder sind flexibel genug zur freien Weiterverbreitung. Diese teilweise antisystemischen Verhaltensweisen sind für Staaten und Unternehmen schwierig zu erfassen und zu kontrollieren. Ihre Lesart als Netzwerke scheint eine bessere Erklärung abzugeben, als der Versuch, sie in die Logik des E-Kapitalismus und dessen algorithmenbasierte Gouvernementalität einzuordnen. Trotz ihrer Inkompatibilität mit dieser Logik handelt es sich um Netzwerke, in denen gearbeitet wird und - zumindest auf kurze Sicht - bestimmte Zielvorstellungen existieren. Teils agieren diese Netzwerke autonom, und teils über Verhandlungen über die von Unternehmen und von öffentlichen Institutionen bedienten Machtstrukturen verwalteten Produkte. Die formelle und informelle Produktion wird also verflochten, genauso wie die elitär legitimierten Kulturen und diejenigen, die aus alternativen symbo- 
lischen Spielereien oder der bildgewaltigen Kreativität der Jugendlichen hervorgehen. Im Fall der chilenischen Studentenbewegung oder den empörten Spaniern wird von der Möglichkeit ausgegangen, dass unabhängige Sektoren zu Produzenten einer "gemeinschaftlichen Kontrolle, einer Art Volksmacht unter einer neuen Bedeutung als Gegengewicht zur Staatlichkeit und dem Wirtschaftsmarkt« werden könnten (Azócar 2014).

Ohne tatsächlich das institutionelle und das politische System zu hacken, ignorieren viele Menschen das System, oder sie kennen es nicht. Lucía Garay betitelte ihre Analyse des argentinischen Schulsystems mit Así ¿quién quiere estar integrado? (dt. »Wer will unter diesen Bedingungen überhaupt integriert werden?«). Die Aussage stammt von einer Jugendlichen und kontrastiert die üblichen Reden von Politikern, Beamten und Pädagogen. Sie beauftragen die Schule mit dem Kunststück, für soziale Inklusion zu sorgen, während, wie die Autorin erklärt, »die Mechanismen des Überlebens und der kollektiven Repräsentation aufgrund der Arbeitslosigkeit, der Arbeitsmarktveränderungen und dem Strukturverlust der sozialen und politischen Organisationen, die ihnen Halt gaben« immer weiter abgebaut worden sind. Sofern diese Rahmenbedingungen nicht erfüllt sind, wird der Auftrag der Schule zu einem Mandat ohne Grundlage, das folglich leichter in Gewalt, folgenschweren Konsequenzen familiärer Misserfolge und entmutigenden Erwartungen mündet.

Die eigene Lebensgestaltung oder eine Alternative fürs Bürgersein zu finden, war in vorrevolutionären Zeiten noch eine Utopie. Nach dem Fall der Berliner Mauer und den jüngsten (neoliberalen) Misserfolgen in Lateinamerika, allen voran in Nicaragua und Venezuela, haben feministische Bewegungen und Initiativen empörter Jugendlicher, enttäuschter Bürger oder Migranten bescheidenere Erwartungen. Die Optionen zur Konfliktlösung ändern sich. Die Minderheit, die vom Tech-Kapitalismus - wie ihn Éric Sadin nennt profitiert, setzt einerseits darauf, die unterschiedlichen sozialen Bewegungen zu unterdrücken oder zu kooptieren. Andererseits ist sie oft auch bereit, auf einige spezifische Forderungen einzugehen, Gesetzesänderungen voranzutreiben und so die bestehende Ordnung anzupassen, um den aufkeimenden sozialen Widerstand einzudämmen.

Aus einigen aufständischen Bewegungen heraus wird versucht, Zugang zur institutionalisierten Macht zu erstreiten: die 15M-Bewegung, aus der Podemos hervorging, oder die Anführer der chilenischen Studentenbewegung haben es geschafft, als Abgeordnete ins Parlament gewählt zu werden. In Mexiko akzeptieren viele Akteure offene Debatten im kommerziellen Fernsehen, damit junge Menschen Bild und Mikrofon zur Verfügung haben. Andere ver- 
suchen hingegen, Wege für alternatives Handeln aufzubauen. Sie arbeiten mit NGOs zusammen, weil sie der Repräsentation von kritischen Stimmen in den klassischen Medien skeptisch gegenüberstehen. Dabei ist ihnen wohl bewusst, dass die Medien die wirtschaftliche Prekarität der jungen Generation oft gar nicht erwähnen. Den Tausenden von getöteten oder verschwundenen Jugendlichen geben sie entweder kaum Raum, oder sie verzerren die schrecklichen Ereignisse.

Angesichts geschwächter Institutionen reichen Bewegungen mit Überraschungseffekt oder die virtuelle virale Ausbreitung von Protesten im Internet nicht mehr aus. Solche Bewegungen sind ebenso prekär wie die Lebensrealität ihrer Vertreter. Welche gesellschaftlichen Alternativen oder neue Formen der Staatsbürgerschaft gibt es inmitten der vom Neoliberalismus und einigen sozial destruktiven Strömungen des E-Kapitalismus geschwächten Nationalstaaten? Der stärkste Rückgang bei der Wahlbeteiligung wird in der jungen Generation beobachtet. Das wachsende Misstrauen in die Parteien wird weder durch vereinzelte Proteste, noch durch gemeinsame Gegenbewegungen im Internet aufgelöst. Mit den Ergebnissen der Nationalen Jugendumfrage in Mexiko aus dem Jahr 2005 kamen alarmierende Ergebnisse über den Zusammenhang zwischen Informalität und Illegalität bei Jugendlichen ans Licht. Rossana Reguillo stellte fest, dass junge Menschen sich schon lange nicht mehr für Parteien, sondern für die Ursachen der Missstände interessieren. Die junge Generation und andere empörte Mitmenschen handeln und mobilisieren sich heute eher aufgrund von folgenschweren Ereignissen, anstatt durch deren Ursachen: Bei Protestmärschen und Zusammenkünften in digitalen Netzwerken fällt uns ein Übergang vom Gefühl der Empörung zu einer greifbaren Politik schwer. In einigen Fällen sind die Ursachen für die Missstände und Unzufriedenheit wiederum langanhaltender und nachhaltiger, wie im Falle der verschiedenen Feminismen.

Man geht also davon aus, dass eine steigende Anzahl unzufriedener Menschen mit einem Gefühl der Hoffnungslosigkeit gegenüber einem systemischen Wandel immer näher an die Option rückt, die bestehenden Strukturen zu hacken oder zu stürzen. Dies geschieht entweder über einen »leichten Hack«, das heißt durch Ignoranz und Unwissenheit, oder durch aufrührerisches Verhalten, bewusste Störaktionen. Außerdem existieren die Praxis der kostenfreien Downloads zur Informationserlangung, sowie der nichtsystemische oder informelle Gebrauch verschiedener Ressourcen zum eigenen Vorteil, beziehungsweise zum Vorteil so genannter alternativer Mikrogemeinschaften. Cuauhtémoc Medina, ein in Mexiko bekannter Kritiker und Kura- 
tor, veröffentlichte vor kurzem ein neues Buch mit dem Titel Abusos mutuos (dt. gegenseitige Missbräuche). Die in solchen alternativen Gemeinschaften entwickelte Idee der Commons, also selbstorganisiert geteilter und bedürfnisorientierter Allgemeingüter, hat zu einer Milderung der Stigmatisierung der Piraterie geführt. Der Begriff hat andere Arten der Wissensproduktion- und Verbreitung etabliert, die nicht auf dem Profit einiger weniger gründen, sondern von einem gemeinschaftlich geteilten Wert der Güter ausgehen. Doch wer teilt sich diese Güter? Wo kommt das Gemeinschaftliche an seine Grenzen? Bei jedem Hackangriff geht es um die Missachtung einer bestehenden Ordnung oder eines vorgegebenen Vorgangs. Wer und wie viele entscheiden in der heutigen Gesellschaft also, was »das Gemeinschaftliche« ist, wo es beginnt, und wo das Geteilte seine Grenzen hat?

Die Skepsis gegenüber »legalen« Wirtschafts- und Kommunikationssystemen, sowie deren nichtsystemischer Gebrauch sind nicht nur als Akt der Rebellion oder als Kritik zu bewerten, sondern auch als eine Ressource der Konservativen. Beispiele sind der Trumpismus in den USA, Vox in Spanien, die italienische Fünf-Sterne-Bewegung, Unterstützergruppen von Marine Le Pen und anderen Politikern aus Osteuropa, Bolsonaro in Brasilien und die neuen Rechtsextremen in Costa Rica und Uruguay, zwei der demokratischsten Länder Lateinamerikas. Sie alle nutzen die soziodigitalen Netzwerke zur Polarisierung der Bürger. Täglich verbreiten sie Fake-Daten und Falschinterpretationen. Unterbewertete Themen oder Angelegenheiten, die die Kapazitäten der Regierung überschreiten (Migration, Gewalt etc.), werden durch sie Teil der nationalen Politik. In Verbindung mit einer rückwärtsgewandten moralischen Besessenheit gegenüber Themen wie der Ehe für Alle, der Abtreibung und einem gewissen Nativismus präsentieren sie sich als die einzigen Vertreter mit der Fähigkeit und der Bereitschaft, sich allem zu stellen. Eine Entwicklung, die beunruhigt. In internationalen Gremien hat sich die extreme Rechte der genannten Länder trotz ihrer Unterschiede zusammengeschlossen und setzt sich für ihre Themen im Sinne einer neuen politischen Agenda ein. Darunter fällt etwa die kollektive Beschuldigung der »Ausländer« mit besonderem Fokus auf Muslime, oder, in anderen Fällen, Afrikaner oder Lateinamerikaner, die ihre Heimatländer aus wirtschaftlicher Not, politischer und sozialer Verfolgung und unter verschiedensten Umständen und Ausmaßen verlassen. Die gleichen Arten von Webseiten, Tweet-Stilen und der ideologischen Voreingenommenheit wirken als eine Art gemeinsame Vorlage. Sie tritt beispielsweise auf entsprechenden italienischen Portalen, Seiten von 
Bolsonaro-Anhängern in Brasilien und in den Foren der Trump-Unterstützer in Erscheinung, darunter CitizenGo (Applebaum 2019, 2-5).

Sowohl die Presse und das Fernsehen, als auch von ehemaligen Nutzern traditioneller Medien frequentierte alternative Netzwerke haben Schwierigkeiten, eine Falschmeldung von einer wahren Begebenheit zu unterscheiden. Ein Beispiel ist der Brand der Notre Dame in Paris: Angeblich hatte man auf der Straße feiernde Muslime gesehen, und neben Fotos der brennenden Kathedrale kursierten Gerüchte über einen schon existierenden Plan für den Bau einer Moschee an der gleichen Stelle. In Brasilien gingen Tweets und Videos um, die Lehrer beschuldigten, ihre Schüler zu einer »Verwirrung« der Gesellschaft zu verführen. Der Effekt dieser Meldungen ist umso intensiver, wenn Führungsfiguren wie Santiago Abascal von Vox oder Bolsonaro diese Sichtweisen retweeten und den Zorn der unzufriedenen Bürger dadurch antreiben.

Judith Butler und Isabel Lorey finden Korrelationen zwischen den hackerähnlichen Netzpraktiken der Rechten und der Prekarität: Prekarisierung ist demnach ein Instrument der Regierung und der Selbstverwaltung der Subjekte (Lorey 2016). Die fortwährenden Verfechter einer kohäsiven Gesellschaftsordnung im liberalen Sinne fühlten sich von solchen Bewegungen nicht angesprochen, solange sie nicht zu politischen Parteien wurden. Zunehmend organisieren sie sich aber als Partei und gewinnen immer häufiger Wahlen. Aus der Sicht des Neoliberalismus beeinträchtigt etwas Unvorhersehbares oder etwas zufällig Mögliches, sei es bei den Protestbewegungen oder beim Zerfall der politischen Parteien, den nationalen und internationalen wirtschaftlichen Wettbewerb nicht: Die gesunde US-Wirtschaft in der Amtszeit von Trump scheint diese Ansicht zu bestätigen. Angesichts der wirtschaftlichen Einstürze in Argentinien während der Macri-Regierung oder der Rezession in Kolumbien und Brasilien ergibt sich jedoch ein anderes Bild. Möglicherweise verliert Butlers und Loreys Argument über die Prekarisierung als Regierungsressource damit nicht seine Gültigkeit, aber muss für den lateinamerikanischen Kontext anhand anderer Variablen neu gedacht werden.

Der nigerianische Schriftsteller und Historiker Teju Cole beschreibt die maschinelle Anpassung und das Hacking wie folgt:

P. Sie besitzen die doppelte Staatsbürgerschaft. Was denken Sie als Amerikaner über ein politisches System, das sowohl Persönlichkeiten wie Trump, als auch Obama hervorbringen kann?

$R$. Obama war ein Produkt dessen, was in der Informationstechnologie 
als »maschinelles Lernen« bekannt ist. Das politische System der USAmerikaner arbeitet wie diese Maschinen. Mit der Zeit wurde es präzisiert, und brachte zu einem Zeitpunkt jemanden wie Obama hervor: ein großer, gutaussehender und wortgewandter schwarzer Mann, dessen politische Vision ganz eng mit dem Traum des amerikanischen Imperialismus zusammenhängt. Das Problem dabei ist, dass, wie bei jedem Computersystem, ein Hacker auftauchen kann, der den Schwachpunkt der Maschine kennt und sie in die Luft jagt. Trump wusste genau, dass der Schwachpunkt des Systems Obama das Ressentiment der weißen Bevölkerung war. Schwarze Menschen sind natürlich schlechter dran, aber das spielte dabei keine Rolle. Hinzu kommt seine Fähigkeit, sich der Kommunikationsmedien zu bedienen, die es nicht schaffen, eine Narrative zu generieren, sondern bloß eine bereits existierende zu verstärken. Sie funktionieren wie ein Lautsprecher, und sind deshalb unethisch. Sie erhöhen lediglich die Lautstärke eines Signals, das sie erhalten (Lago 2016). 